\title{
Reliable on-line re-optimization control of a fed-batch fermentation process using bootstrap aggregated extreme learning machine
}

\author{
Carolina Maria Cardona Baron and Jie Zhang \\ School of Engineering, Merz Court, Newcastle University, Newcastle upon Tyne NE1 7RU, \\ UK \\ jie.zhang@newcastle.ac.uk
}

\begin{abstract}
This paper presents a reliable on-line re-optimization control of a fedbatch fermentation process using bootstrap aggregated extreme learning machine. In order to overcome the difficulty in developing detailed mechanistic models, extreme learning machine (ELM) based data driven models are developed. In building an ELM model, the hidden layer weights are randomly assigned and the output lay er weights are obtained in a one step regression ty pe of learning. This feature makes the development of ELM very fast. A single ELM model can lack of robustness due the randomly assigned hidden layer weights. To overcome this problem, multiple ELM models are developed from bootstrap re-sampling replications of the original training data and are then combined. In addition to enhanced model accuracy, bootstrap aggregated ELM can also give model prediction confidence bounds. A reliable optimal control policy is achieved by means of the inclusion of model prediction confidence bounds within the optimization objective function to penalize wide model prediction confidence bounds which are associated with uncertain predictions as a consequence of plant modelmismatch. Finally, in order to deal with unknown process disturbances, an online re-optimization control strategy is developed in that on-line optimization is carried out while the batch process is progression. The proposed technique is successfully implemented on a simulated fed-batch fermentation process.
\end{abstract}

Keywords: Fed-batch processes, Fermentation, Neural networks, Extreme learning machine, Re-optimization.

\section{Introduction}

Fermentation is an important processing step in biochemical and pharmaceutical industry and is generally carried out in batch or fed-batch mode. In general, common products such as antibiotics, proteins, food cultures, and more, are manufactured through bioprocessing techniques [1]. Nowadays, the biochemical industry has been developed through modern and sophisticated technologies, specially designed for process contro 1 and automation purposes. Coupled with an increase in the demand of products and quality requirements, competitive markets have arisen not only on a local scale, but also worldwide. Hence, the optimization of biochemical processes becomes increasingly 
more important. However, the biotechnological process such as fermentation process is difficult to control, in as much as the sensibility of the micro-organism demands accurate control strategies. Thus enhancing fermentation process operation is an important means to improve product quality and process profit. The massive consumption of biochemical products results in a competitive market, making the maximization of biomass production a key target to be achieved. The complexity of the fermentation processes dynamics makes this a non-trivial and challenging but also very interesting optimization problem.

For the optimal control of biochemical processes, which are typically highly nonlinear systems, accurate models capable of providing good long range predictions are required. Due to the complex dynamics of the growing microorganisms in biochemical processes, mechanistic models are usually very difficult to be developed. To overcome this problem, data-driven modelling techniques based on process operation data have been recently developed to provide accurate solutions for process modelling $[2,3]$. However, the collection of process operational data is typically limited partly because of the high costs involved in the experiments for data acquirement, coupled with the physical limitation to measure certain key process variables.

In the past a few years, neural networks have been widely accepted as an effective way to build accurate data-driven models from process operation data [4-7]. The main advantage of neural networks is their ability to model complex non-linear processes by learning the nonlinear relationship between model inputs and outputs from the process operation data. This is perhaps achieved through their parallel structure that provides them with excellent capabilities to store knowledge. Artificial neural networks resembles the human brain in the sense that knowledge is learnt from observations and stored in the form of inter-neuron connection strengths [8].

A common problem of neural networks is the over fitting of the training data leading to poor generalization capabilities in that the neural network model gives excellent performance on the training data but considerably large prediction errors on unseen validation data. The speed of conventional neural network training is also a concern as training with the traditional backpropagation training algorithm is typically very slow due to the iterative procedure. In order to address those common drawbacks with conventional neural networks, a novel algorithm developed by Huang et al. [9], called Extreme Learning Machine (ELM), has been shown to be able to provide an extremely fast learning speed and better generalization capabilities in comparis on to traditional learning algorithms. In ELM hidden layer weights are randomly chosen and the output layer weights are obtained in a one step regression like approach. This significantly reduces the computational efforts in neural network training. However, single ELM models can lack robustness and give varying performance due to random nature in assigning the hidden layer weights. To address this is sue, the idea of bootstrap aggregated neural networks [10] can be used in developing bootstrap aggregated ELM. The use of bootstrap aggregated neural networks is widely recognized as an effective method to improve the robustness of neural network models and enhance the model generalization capabilities [11-15].

Zhang [16] proposed a reliable optimization strategy based on bootstrap aggregated neural network models, where a reliable optimal control policy is obtained by means of penalizing wide model prediction confidence bounds within the objective function of the optimization problem. The modified optimization objective function penalizes wide 
model prediction confidence bounds. By such a means, the obtained optimal control policy can be successfully implemented in the actual process without suffering from performance degradation, which is commonly caused by plant-model mismatches.

The paper is organized as follows. A feed-batch fermentation process is presented Section 2. Section 3 introduces the proposed bootstrap aggregated ELM. Section 4 presents the modelling of the feed-batch fermentation process using bootstrap aggregated ELM. Reliable optimization control of the feed-batch fermentation process is presented Section 5. Both off-line optimization and on-line re-optimization control are presented. Finally, Section 6 draws some concluding remarks.

\section{A Fed-Batch Fermentation Process}

The fed-batch fermentation process considered in this paper uses Baker's yeast as the basis reactant. The kinetic and dynamic model is taken from [17] and includes a dynamic model based on mass balance equations for glucose, ethanol, oxygen and biomass concentrations. The kinetic model contains the following equations [17]:

Glucose uptake rate:

$$
Q_{s}=Q_{s, \max } \frac{C_{s}}{K_{s}+C_{s}}\left(1-e^{-t / t_{d}}\right)
$$

Oxidation capacity:

$$
Q_{o, \lim }=Q_{o, \max } \frac{C_{o}}{K_{o}+C_{o}} \frac{K_{i}}{K_{i}+C_{e}}
$$

Specific growth rate limit:

$$
Q_{s, l i m}=\frac{\mu_{c r}}{Y_{x / s}^{o x}}
$$

Oxidative glucose metabolism:

$$
Q_{s, o x}=\min \left(\begin{array}{c}
Q_{s} \\
Q_{s, l i m} \\
Q_{o, l i m} / Y_{o / s}
\end{array}\right)
$$

Reductive glucose metabolism:

$$
Q_{s, \text { red }}=Q_{s}-Q_{s, o x}
$$

Ethanol uptake rate:

$$
Q_{e, u p}=Q_{e, \max } \frac{C_{e}}{K_{e}+C_{e}} \frac{K_{i}}{K_{i}+C_{e}}
$$

Oxidative ethanol metabolism:

$$
Q_{e, o x}=\min \left(\begin{array}{c}
Q_{e, u p} \\
\left(Q_{o, l i m}-Q_{s, o x} Y_{o / s}\right) Y_{e / o}
\end{array}\right)
$$


Ethanol production rate:

$$
Q_{e, p r}=Q_{s, r e d} Y_{e / s}
$$

Total specific growth rate:

$$
\mu=Q_{s, o x} Y_{x / s}^{o x}+Q_{s, r e d} Y_{x / s}^{r e d}+Q_{e, o x} Y_{x / e}
$$

Carbon dioxide production rate:

$$
Q_{c}=Q_{s, o x} Y_{c / s}^{o x}+Q_{s, r e d} Y_{c / s}^{r e d}+Q_{e, o x} Y_{c / e}
$$

Oxygen consumption rate:

$$
Q_{o}=Q_{s, o x} Y_{o / s}+Q_{e, o x} Y_{o / e}
$$

Respiratory Quotient:

$$
R Q=Q_{c} / Q_{o}
$$

The mass balance equations describe the dynamic of glucose, ethanol, oxygen and biomass concentrations as follows [17]:

$$
\begin{aligned}
& \frac{d C_{s}}{d t}=\frac{F}{V}\left(S_{o}-C_{s}\right)-\left(\frac{\mu}{Y_{x / s}^{o x}}+\frac{Q_{e, p r}}{Y_{e / s}}+Q_{m}\right) C_{x} \\
& \frac{d C_{o}}{d t}=-Q_{o} C_{x}+k_{L} a_{o}\left(C_{o}^{*}-C_{o}\right)-\frac{F}{V} C_{o} \\
& \frac{d C_{e}}{d t}=\left(Q_{e, p r}-Q_{e, o x}\right) C_{x}-\frac{F}{V} C_{e} \\
& \frac{d C_{x}}{d t}=\mu C_{x}-\frac{F}{V} C_{x} \\
& \frac{d V}{d t}=F \\
& k_{L} a_{o}=113\left(\frac{F_{a}}{A_{R}}\right)^{0.25}
\end{aligned}
$$

where $C_{s}, C_{o}, C_{e}$, and $C_{x}$ represent, respectively, the concentrations of glucose, oxygen, ethanol and biomass, $F$ and $F_{a}$ stand for feed rate and air feed rate respectively, and $A_{R}$ denotes the cross-sectional area of the reactor. The other symbols and values of model parameters are given in Tables 1 and 2 .

Based on the mechanistic model, a simulation program is developed in MATLAB [18]. The simulation program is used to generate process operational data and to test the developed models and optimization control policies. The batch initial conditions considered for the simulation are taken from [17] and are summarized as follows:

- Initial conditions: $C_{s}(0)=7 \mathrm{~g} \mathrm{~L}^{-1} ; C_{o}(0)=7.8 e^{-3} \mathrm{~g} \mathrm{~L}^{-1} ; C_{e}(0)=0 \mathrm{~g} \mathrm{~L}^{-1}$; $C_{x}(0)=15 \mathrm{~g} \mathrm{~L}^{-1} ; V(0)=50000 \mathrm{~L}$

- Volume of the fermentor $\mathrm{V}_{\mathrm{f}}=100 \mathrm{~m}^{3}$

- Concentration of feed $\mathrm{S}_{0}=325 \mathrm{~g}$ glucose $\mathrm{L}^{-1}$

- Final time: $\mathrm{t}_{\mathrm{f}}=16.5 \mathrm{~h}$ 
Table 1. Definition of process variables and parameters.

\begin{tabular}{llll}
\hline variables & definitions & $\begin{array}{l}\text { Superscripts } \\
\text { and subscripts }\end{array}$ & definitions \\
\hline $\mathrm{k}_{\mathrm{L} a O}$ & total volumetric mass transfer coefficient $\left(\mathrm{h}^{-1}\right)$ & $*$ & interface \\
$\mathrm{K}_{\mathrm{e}}$ & saturation constant for ethanol $\left(\mathrm{gL}^{-1}\right)$ & $\mathrm{cr}$ & critic \\
$\mathrm{K}_{\mathrm{i}}$ & inhibition constant $\left(\mathrm{gL}^{-1}\right)$ & $\mathrm{e}$ & ethanol \\
$\mathrm{K}_{\mathrm{o}}$ & saturation constant for oxy gen $\left(\mathrm{gL}^{-1}\right)$ & $\mathrm{lim}$ & limitation \\
$\mathrm{K}_{\mathrm{s}}$ & saturation constant for substrate $\left(\mathrm{gL}^{-1}\right)$ & $\mathrm{o}$ & oxygen \\
$\mathrm{Y}_{\mathrm{i} / \mathrm{j}}$ & yield of component $i$ on $j\left(\mathrm{gg}^{-1}\right)$ & ox & oxidative \\
$\mathrm{V}$ & volume $(\mathrm{L})$ & $\mathrm{pr}$ & production \\
$\mu$ & specific growth rate $\left(\mathrm{h}^{-1}\right)$ & red & reductive \\
& & $\mathrm{s}$ & substrate \\
& & & glucose) \\
& & up & uptake \\
& & $\mathrm{x}$ & biomass \\
\hline
\end{tabular}

Table 2. Numeric values of the parameters in the fed-batch model [18].

\begin{tabular}{cccc}
\hline parameters & values & parameters & values \\
\hline $\mathrm{K}_{\mathrm{e}}$ & $0.1 \mathrm{gL}^{-1}$ & Yx/e & $0.7187 \mathrm{gg}^{-1}$ \\
$\mathrm{~K}_{\mathrm{i}}$ & $3.5 \mathrm{gL}^{-1}$ & Qe,max & $0.238 \mathrm{gg}^{-1} \mathrm{~h}^{-1}$ \\
$\mathrm{~K}_{\mathrm{o}}$ & $9.6 \times 10^{-5} \mathrm{gL}^{-1}$ & Qo,max & $0.255 \mathrm{gg}^{-1} \mathrm{~h}^{-1}$ \\
$\mathrm{~K}_{\mathrm{s}}$ & $0.612 \mathrm{gL}^{-1}$ & Qs,max & $2.943 \mathrm{gg}^{-1} \mathrm{~h}^{-1}$ \\
$Y_{X / S}^{O X}$ & $0.585 \mathrm{gg}^{-1}$ & $\mathrm{Q}_{\mathrm{m}}$ & $0.03 \mathrm{gg}^{-1} \mathrm{~h}^{-1}$ \\
$Y_{X / S}^{\text {red }}$ & $0.05 \mathrm{gg}^{-1}$ & $\mathrm{~S}_{\mathrm{o}}$ & $325 \mathrm{gh}^{-1}$ \\
$\mathrm{Y}_{\mathrm{o} / \mathrm{s}}$ & $0.3857 \mathrm{gg}^{-1}$ & & \\
$\mathrm{Y}_{\mathrm{o} / \mathrm{e}}$ & $0.8904 \mathrm{gg}^{-1}$ & $C_{o}^{*}$ & $0.006 \mathrm{gh}^{-1}$ \\
$\mathrm{Y}_{\mathrm{e} / \mathrm{s}}$ & $0.4859 \mathrm{gg}^{-1}$ & $\mathrm{~A}_{\mathrm{R}}$ & $12.56 \mathrm{~m}^{2}$ \\
$\mathrm{Y}_{\mathrm{e} / \mathrm{o}}$ & $1.1236 \mathrm{gg}^{-1}$ & $\mu_{\mathrm{cr}}$ & $0.21 \mathrm{~h}^{-1}$ \\
\hline
\end{tabular}

\section{Bootstrap Aggregated Extreme Learning Machine}

\subsection{Extreme Learning Machine}

Fig. 1 shows a typical feedforward neural network, which can be used to model complex nonlinear systems and is capable of representing the nonlinear relationships between process input and output variables by learning from the training data presented 
to the network using a network training algorithm. Although the development of neural network models is significantly more practical and easier to implement than the classical mathematical modelling approaches based on first principles, the time required to train neural networks using traditional network training algorithms is considerably high, making the neural network training a slow process [9].

Many researchers have worked on enhancing the generalization capabilities of neural networks. However, most of the neural network training methods still involve the tuning of all network weights and biases, and in many cases, still require iterative computations until convergence or a pre-defined maximum iterations has been reached. To reduce the computational burden in neural network training, Huang et al. [9] propose the ELM, a novel learning algorithm for single hidden layer feedforward networks (SLFN). In ELM, some of the network parameters are chosen randomly and this significantly reduces the training time while achieving good generalization capabilities.

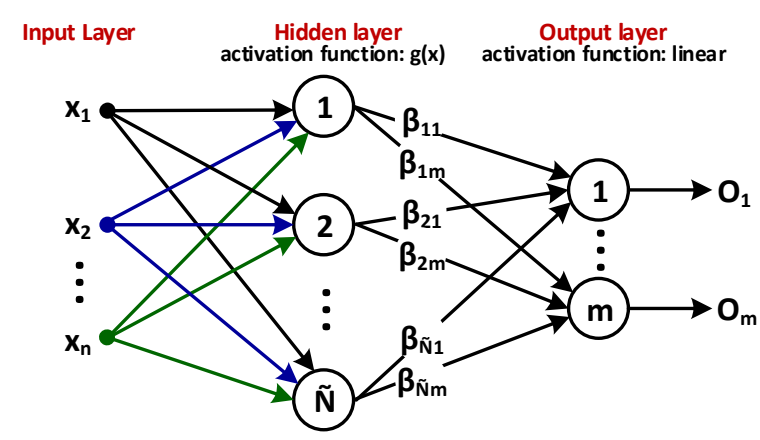

Fig.1. A single hidden layer feedforward network [18].

In the ELM algorithm proposed by Huang et al. [9], a SLFN is built from $N$ distinct pair of samples $\left(x_{i}, t_{i}\right)$ with $x_{i}=\left[x_{i 1}, x_{i 2}, \ldots, x_{i n}\right]^{T} \in R^{n}$ and $t_{i}=\left[t_{i 1}, t_{i 2}, \ldots, t_{i m}\right]^{T} \in$ $R^{m}$. Let this SLFN have $\tilde{\mathrm{N}}$ hidden neurons. The $i$ th neuron in the hidden layer is connected with the input layer through a weighting vector, $w_{i}=\left[w_{i 1}, w_{i 2}, \ldots, w_{i n}\right]^{T}$, and has activation function $g(x)$ and bias $b_{i}$. The nodes in the output layer are connected to the $i$ th hidden neuron through the weighting vector $\beta_{i}=\left[\beta_{i 1}, \beta_{i 2}, \ldots, \beta_{i m}\right]^{T}$, and they use a linear activation function as follows.

$$
\begin{aligned}
& \sum_{i=1}^{\tilde{\mathrm{N}}} \beta_{i} g_{i}\left(x_{j}\right)=\sum_{i=1}^{\tilde{\mathrm{N}}} \beta_{i} g\left(w_{i} \cdot x_{j}+b_{i}\right)=o_{j} \\
& \quad j=1,2, \ldots, N
\end{aligned}
$$

As long as $g(x)$ is infinitely differentiable, the SLFN can learn $N$ distinct observations, which can be written as $\sum_{j=1}^{\tilde{\mathrm{N}}}\left\|o_{j}-t_{j}\right\|=0$. Then there should exist finite values of $\beta_{i}, w_{i}$, and $b_{i}$ that can meet the following:

$$
\sum_{i=1}^{\tilde{\mathrm{N}}} \beta_{i} g\left(w_{i} \cdot x_{j}+b_{i}\right)=t_{j}
$$




$$
j=1,2, \ldots, N
$$

The relationship given in $\mathrm{Eq}(20)$ can be written in matrix notation as $\mathrm{Eq}(21)$, where $\boldsymbol{H}$ is called the hidden layer output matrix:

$$
\begin{aligned}
& \boldsymbol{H} \beta=\boldsymbol{T} \\
& H\left(w_{1}, \ldots, w_{\tilde{\mathrm{N}}}, b_{1}, \ldots, b_{\tilde{\mathrm{N}}}, x_{1}, \ldots, x_{N}\right)= \\
& {\left[\begin{array}{ccc}
g\left(w_{1} \cdot x_{1}+b_{1}\right) & \cdots & g\left(w_{\tilde{\mathrm{N}}} \cdot x_{1}+b_{\tilde{\mathrm{N}}}\right) \\
\vdots & \ddots & \vdots \\
g\left(w_{1} \cdot x_{N}+b_{1}\right) & \cdots & g\left(w_{\tilde{\mathrm{N}}} \cdot x_{N}+b_{\tilde{\mathrm{N}}}\right)
\end{array}\right]_{N \times \tilde{\mathrm{N}}}} \\
& \beta=\left[\begin{array}{c}
\beta_{1}^{T} \\
\vdots \\
\beta_{\tilde{\mathrm{N}}}^{T}
\end{array}\right]_{\tilde{\mathrm{N}} \times m} \quad \text { and } \quad \boldsymbol{T}=\left[\begin{array}{c}
t_{1}^{T} \\
\vdots \\
t_{N}^{T}
\end{array}\right]_{N \times n}
\end{aligned}
$$

The ELM algorithm proposed in [9] suggests setting the parameters $\beta_{i}$ and $w_{i}$ randomly and compute the matrix $\boldsymbol{H}$. Then the remaining unknown variable in $\mathrm{Eq}(21), \beta$, can be calculated as:

$$
\hat{\beta}=\boldsymbol{H}^{\dagger} \boldsymbol{T}
$$

In the above equation, $\boldsymbol{H}^{\dagger}$ corresponds to the Moore-Penrose generalized inverse of the matrix $\boldsymbol{H}$, which can be found through several methods such as the orthogonal projection, singular value decomposition (SVD), orthogonalization method, and iterative method. The last two methods are avoided since iterations are undesired as they can increase the computation times of the ELM algorithm. Instead, if $H^{T} H$ is non-singular, the orthogonal projection method can be used, so $\boldsymbol{H}^{\dagger}=\left(H^{T} H\right)^{-1} H^{T}$. As in many cases the matrix $H^{T} H$ tends to be singular, the SVD method generally performs well under those circumstances.

\subsection{Bootstrap Aggregated ELM}

Regarding to the techniques for developing multiple neural networks, Noor et al. [8] identifies three basic kinds of stacked neural networks where individual networks are combined using a particular method. The first type is the multiple model neural networks, characterized for using different training data to build the individual networks. The training data can relate to different inputs and include information about a wider process operation region. This approach also allows different training algorithms to be used for each individual neural network. On the other hand, the second category employs the same data to train the individual networks, but the data are re-sampled or divided using one of the following methods: bootstrap re-sampling [19], adaboost, "adaptive boosting", or randomization. Finally, the third category involves a selective combination of neural networks in order to reduce the error induced by those neural networks with poorest generalization capabilities. In this paper, a bootstrap aggregated ELM, known as BA-ELM, is proposed. 
As mentioned in [10], the principle of bootstrap aggregated neural networks, shown in Fig. 2, is to develop several neural networks to model the same relationship and them combining them. By this means, model generalization capability and accuracy can be improved as a result of proper combination of all networks, instead of just selecting the "best" individual neural network. The final output of a BA-ELM, expressed in Eq(25), is a weighted combination of the individual ELM outputs.

$$
f(X)=\sum_{i=1}^{n} w_{i} f_{i}(X)
$$

In the above equation, $f(X)$ is the BA-ELM predictor, $f_{i}(X)$ is the $i$ th ELM, $w_{i}$ is the aggregating weight corresponding to the $i$ th ELM, $X$ is the vector of inputs, and $n$ is the number of individual ELM models. The selection of aggregating weights is fundamental to achieving good performance. In general, the simple approach taking equal weights is usually sufficient to attain enhanced model prediction performance. However, aggregating weights can also be obtained by using principal component regres sion (PCR), since it is less sensitive to highly correlated data, which is the case for the individual network predictions.

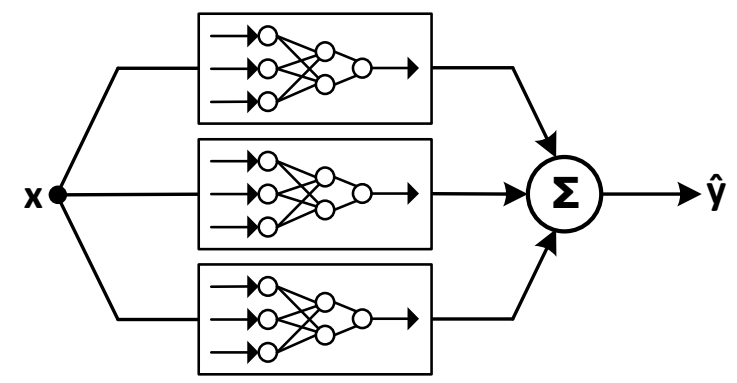

Fig. 2. Bootstrap aggregated ELM [18]

A further advantage in using BA-ELM is that model prediction confidence bounds can be calculated from the individual network predictions as follows [10]:

$$
\sigma_{e}=\left\{\frac{1}{n-1} \sum_{b=1}^{n}\left[y\left(x_{i} ; W^{b}\right)-y\left(x_{i} ; \cdot\right)\right]^{2}\right\}^{1 / 2}
$$

where $\sigma_{e}$ corresponds to the standard error of the $i$ th predicted value, $y\left(x_{i} ; \cdot\right)=$ $\sum_{b=1}^{n} y\left(x_{i} ; W^{b}\right) / n$ and $n$ is the number of ELM models. Under the assumption that prediction errors are normally distributed with zero mean, the $95 \%$ prediction confidence bound can be found as $y\left(x_{i} ; \cdot\right) \pm 1.96 \sigma_{e}$. More reliable predictions are as sociated with small values of $\sigma_{e}$, i.e. narrower confidence bounds. 


\section{$4 \quad$ Process Modelling Using Bootstrap Aggregated ELM}

\subsection{Data generation and pre-processing}

The detailed mechanistic model described in Section 2 is used to generate simulated process operational data. All together 75 batches were simulated with the feed profiles obtained by adding random variations to a base feed profile. The batch duration in divided into 17 equal intervals and the substrate feed rate is kept constant in each interval. Thus the feed profile can be represented by a vector of 17 elements. The developed ELM model is of the following form:

$$
y=f\left(x_{1}, x_{2}, \ldots, x_{17}\right)
$$

where $y$ is the biomass concentration at the end of a batch, $x_{1}$ to $x_{17}$ are the substrate feed rates over a batch.

Data pre-processing is carried out to remove undesired information such as noise, outliers, non-representative samples, etc. Data pre-processing tools include for example normalization to scale the data, filtering to cope with measurement noise, removing trends and outliers to eliminate inconsistent data that potentially will lead to wrong results, etc.

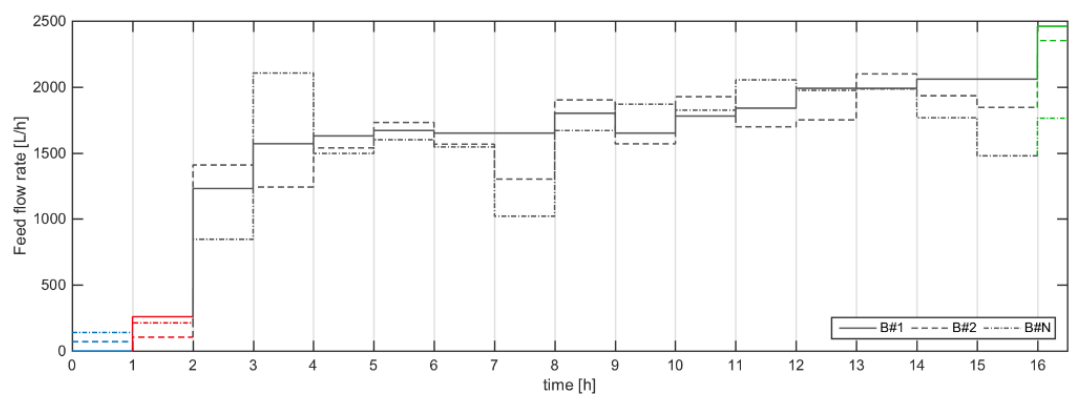

Fig. 3. Normalization, Case A

Normalization of data to zero mean and unit variance is used in this study. As the model inputs are the substrate feed rates at different time stages over a batch, two different cases of normalization are considered here. In Case A, as illustrated in Fig. 3, the substrate feed rate at a particular time is normalized using the local mean and standard deviation at that particular time. In Case B, as illustrated in Fig. 4, the global mean and standard deviation across all the time stages are used.

\subsection{BA-ELM modelling}

Once the data have been scaled to zero mean and unity variance, $80 \%$ (60 batches) of data are randomly selected for model building and the remaining 20\% (15 batches) are used as the unseen validation data. Then, the original training set is here re-sampled using bootstrap re-sampling with replacement [19] to produce $m=50$ different bootstrap replication data sets. On each bootstrap replication data set, an ELM model is developed. The bootstrap re-sampling is a simple technique for generating different 
replications of the original data set in that random samples (batches) from the original data are picked. As a consequence, some samples can be picked more than once and some others may not be picked at all. In this way, the learning data set presented to each ELM is slightly different, which is the powerful concept of BAGNET [10]. As the individual ELM models do not learn exactly the same information, they can complement to each other. The model development procedure is shown in Fig.5.

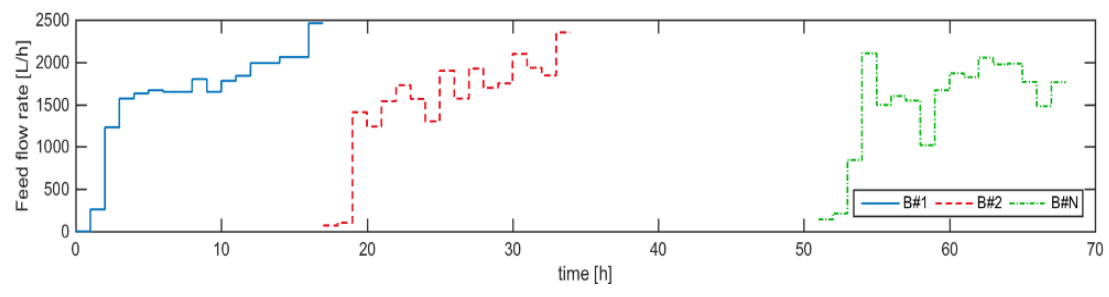

Fig. 4. Normalization, Case B

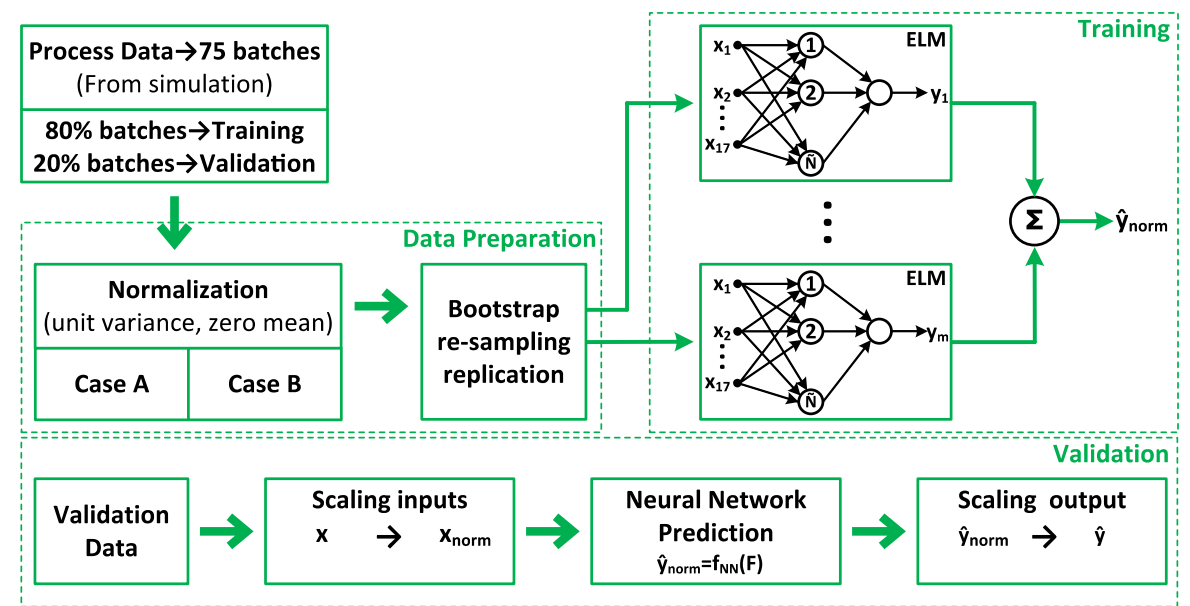

Fig. 5. Model predictions on validation data

Fig. 6 shows model predictions on the 15 unseen batches (validation data) and their respective confidence bounds for the two different cases of normalization, Case A and Case B. Table 3 gives the minimum SSEs for the best individual ELM, stacking 50 ELMs, and stacking 44 ELMs on the training and validation data sets with the two normalization cases. When stacking 44 ELMs, the 6 worst performing ELMs on the training data in the original 50 ELMs are eliminated. It can be seen from Fig. 6 that the model predictions are reasonably accurate. The model with normalization Case A gives better prediction performance as indicated by Fig. 6 and Table 3. Thus, the BA-ELM model with normalization Case A is adopted.

The SSE of the individual networks on training and validation data are shown in Fig. 7. In general, it can be seen from Fig. 7 that network prediction performance on the training data is not always consistent with that on the unseen validation data. It can be 
noticed that an individual network with a small SSE value on the training data can have a large SSE value on the unseen validation data or vice versa. Therefore it is evident that a single neural network is not robust enough to always produce accurate predictions.

Table 3. Minimum SSEs of individual and BA-ELM.

\begin{tabular}{lcccc}
\hline & data & $\begin{array}{c}\text { Best individual } \\
\text { ELM }\end{array}$ & Stacking 50 ELM & Stacking 44 ELM \\
\hline Case A & Training & 0.0767 & 0.0530 & 0.0492 \\
& Validation & 0.1501 & 0.2207 & 0.2191 \\
Case B & Training & $3.05 \mathrm{E}-04$ & $1.81 \mathrm{E}-04$ & $1.75 \mathrm{E}-04$ \\
& Validation & $5.26 \mathrm{E}-04$ & $4.04 \mathrm{E}-04$ & $4.04 \mathrm{E}-04$ \\
\hline
\end{tabular}

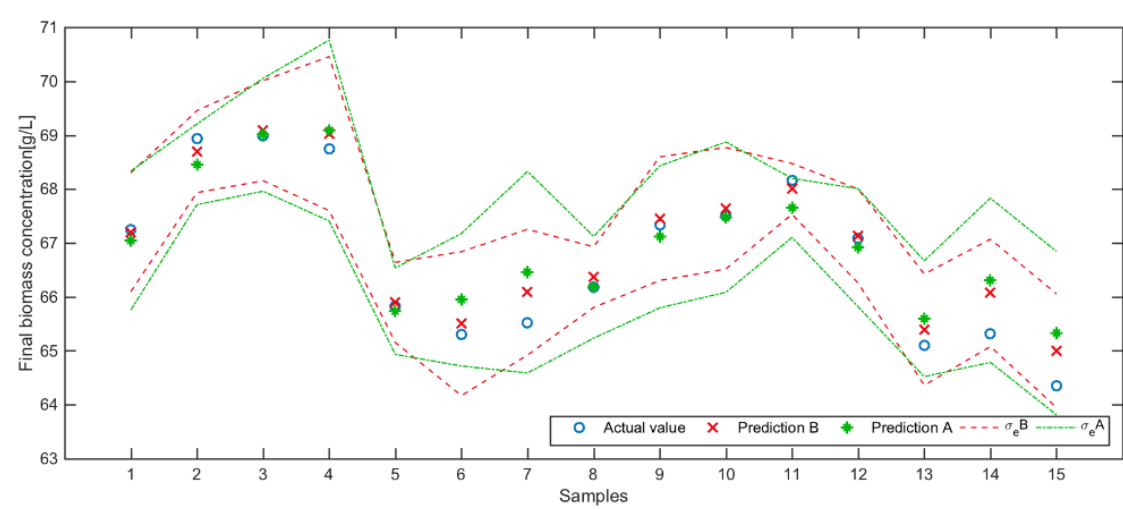

Fig. 6. Model predictions on validation data [18]
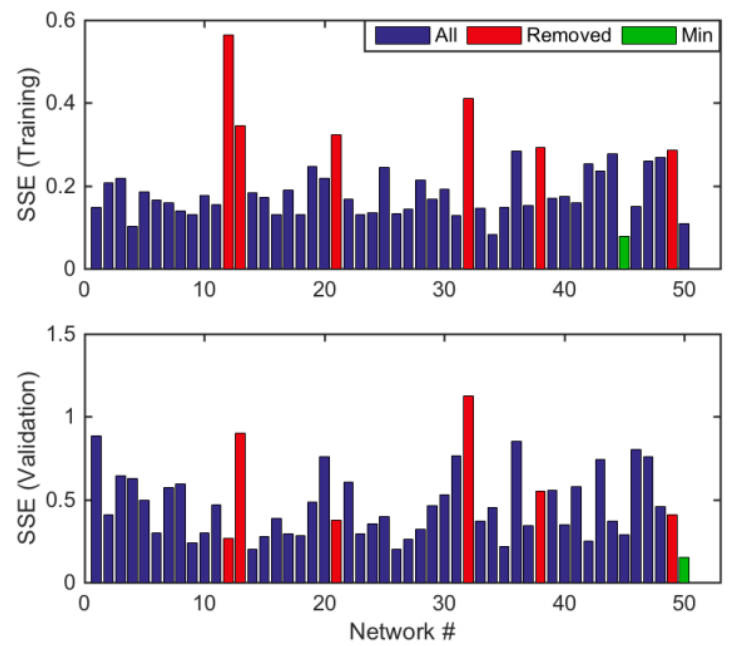

Fig. 7. Model errors of individual networks [18] 
Some individual networks with poor performance on the training data, highlighted in color red in Fig. 7, were removed from the stacked network. Here only 6 such networks are chosen because bad performing on training data does not necessarily imply poor performance on the unseen data. For example, networks \#12 and \#21 are in the worst group based on the performance on the training data. However, they actually have good performance on the unseen validation data. For this reason, it is not advisable to remove a lot of those "poor" performing networks as some of those may give quite good performance on the unseen validation data. Conversely, networks \#13, \#32 and \#38 performed bad in both cases, thus it is appropriate to remove the influence of those networks. Highlighted in color green in Fig. 7, network \#45 gives the minimum SSEs (0.0767) on the training data and network \#50 gives the minimum SSEs (0.1501) on the unseen validation data.
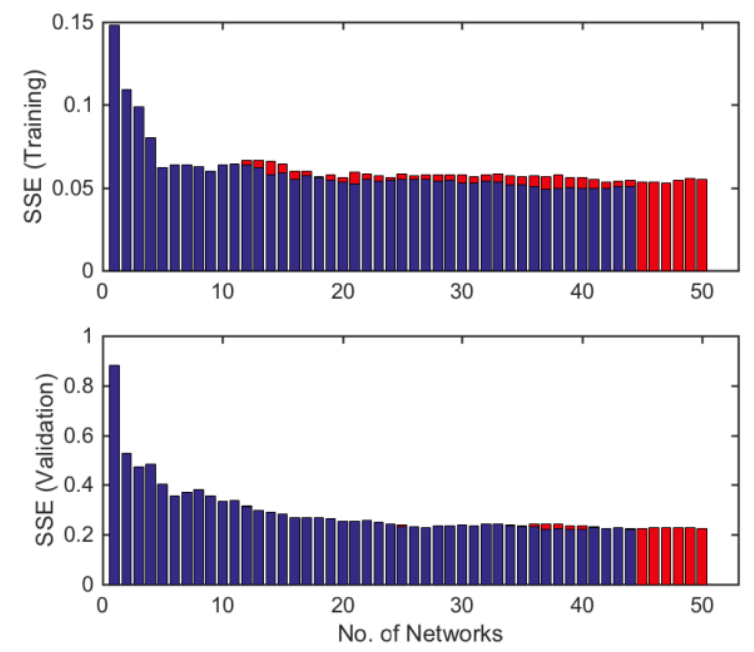

Fig. 8. Model error of stacked networks [18]

In contrast to the performance of individual networks, Fig. 8 clearly shows the advantage of stacking multiple neural networks. The model performance of aggregating different numbers of ELM models, from 1 (the first single ELM) to 50 (aggregating all 50 ELM models), are shown in Fig. 8, which shows the SSE values of BA-ELM with different numbers of ELM models on the training data and the unseen validation data. It can be seen that the highest error in both cases occurs, as is expected, when just one network is used. Then, the errors on both training data and unseen validation data are significantly reduced while more networks are being combined. It is important to notice the consistent pattern of SSE reduction on the training data and on the unseen validation data. Additionally, the influence of the removed networks can be seen in color red, which corresponds to the SSEs before those networks were eliminated. Before removing the worst networks, the minimum SSEs on training data was 0.0530 with the contribution of 47 networks, and 0.2207 for the unseen validation data with 28 networks 
combined. After removing the bad performing networks, the minimum SSE values decrease to 0.0492 with the combination of 37 networks on the training data, and 0.2191 on the unseen validation data with 37 networks combined. On balance, the SSEs were reduced in both training and validation data just by means of combination of multiple non-robust models.

\section{$5 \quad$ Process Optimization Using Bootstrap Aggregated ELM}

\subsection{Off-line optimization}

In this fed-batch fermentation process, the operation objective is to produce as much product as possible subject to operational constraints. The objective function $J$ is formulated using the ELM model. In order to enhance the reliability of optimization, minimizing the width of the model prediction confidence bounds is incorporated in the objective function. The optimization problem can be written as follows:

$$
\begin{gathered}
\min _{F} J=-f_{N N}(F)+\lambda \sigma_{e} \\
\text { subject to: }\left\{\begin{array}{l}
0 \leq F \leq 3000[L / h] \\
V \leq V_{f}=100000[L]
\end{array}\right.
\end{gathered}
$$

where $f_{N N}(F)$ is the BAGNET output, which specifically corresponds to the predicted biomass concentration at the end of the batch $C_{x}\left(t_{f}\right), F=\left[f_{1}, f_{2}, \ldots, f_{17}\right]$ is the vector of substrate feed rates divided in hourly intervals; $\sigma_{e}$ is the standard error of model prediction, and $\lambda$ is a penalty factor for $\sigma_{e}$. Operational constraints are imposed, for instance, the feed flow rate is bounded to maximu $3000[\mathrm{~L} / \mathrm{h}]$ and the volume of the total biomass is restricted by the fermenter volume $V_{f}$. This objective function is aimed to maximise the amount of product while minimise the width of the model prediction confidence bounds to achieve a reliable optimal control policy.

The optimization problem given in $\mathrm{Eq}(28)$ was solved using the Interior-Point algorithm, available in Matlab ${ }^{\circledR}$ Optimization Toolbox, which is an effective non-linear programming method, especially for constrained problems .

Different values of $\lambda$ were considered, in order to analyse the effect of penalising wide model prediction confidence bounds. The optimal control policies obtained for all the cases were tested the mechanistic model based simulation. Table 4 presents the simulation results. The first row in Table 4 corresponds to $\lambda=0$, which is equivalent to the optimization problem without considering the confidence bounds in the objective function. In that case, the neural network prediction for the final biomass is 75.788 $[\mathrm{g} / \mathrm{L}]$ while the actual value (from mechanistic model) is significantly lower at 51.583 $[\mathrm{g} / \mathrm{L}]$, and $\sigma_{e}=0.182$. The notable difference between the aggregated ELM model prediction and the actual value is in fact what motivated the researchers to penalize wide confidence bounds in the objective function [16, 20,21].

The value of $\lambda$ is gradually increased in order to analyse the effect of the penalization term in the objective function. It can be seen from Table 4 that with $\lambda=1$ a considerable improvement of the actual value of biomass is achieved $(59.544[\mathrm{~g} / \mathrm{L}])$, accompanied with the reduction of $\sigma_{e}$ to 0.167 . After trying with further values of $\lambda$, the 
actual final biomass concentration reached $71.236[\mathrm{~g} / \mathrm{L}]$ when $\lambda=12$, and the confidence bounds were reduced to half its initial value; at the same time, the neural network prediction decrease to 75.409 [g/L]. Therefore, from Table 4 it is possible to appreciate that by means of increasing the penalization of wide model prediction confidence bounds, the optimal substrate feeding profile becomes more reliable, since the performance on the actual process is not degraded.

Table 4. Final biomass concentrations and $\boldsymbol{\sigma}_{\boldsymbol{e}}$ with respect to $\lambda[18]$.

\begin{tabular}{cccc}
\hline$\lambda$ & Mechanistic model & BA-ELM & $\sigma_{e}$ \\
\hline 0 & 51.583 & 75.788 & 0.182 \\
1 & 59.544 & 75.781 & 0.167 \\
2 & 65.733 & 75.763 & 0.155 \\
3 & 68.959 & 75.739 & 0.145 \\
5 & 70.203 & 75.682 & 0.130 \\
6 & 70.521 & 75.650 & 0.125 \\
9 & 71.163 & 75.520 & 0.107 \\
12 & 71.236 & 75.409 & 0.096 \\
45 & 71.163 & 74.549 & 0.059 \\
120 & 70.943 & 72.702 & 0.036 \\
\hline
\end{tabular}

However, as the value of $\lambda$ increases, the objective function, $\operatorname{Eq}(28)$, gives more importance to the reduction of $\sigma_{e}$, which as a consequence, will sacrifice the maximization of the final product concentration. Therefore, there is an inherently trade off between the two terms in the objective function. To make this point clear, further higher values of $\lambda$ were tried, corresponding to the last two rows of Table 4 . For $\lambda=120$, the value of $\sigma_{e}$ was notably reduced as well as the relative error between the model prediction and the actual value. However, this was achieved with a reduction in the final biomass production. For this reason, the value of $\lambda=12$ is selected as the optimal weighting factor, since it offers a balance between both objectives .

Fig. 9 presents the optimal feeding profiles corresponding to the "unreliable" optimal control policy when $\lambda=0$ (continuous blue line) and the improved one when $\lambda=12$ (red dashed line). Fig. 10 and Fig. 11 show the profiles of biomass, glucose, oxygen, ethanol and volume when the optimal substrate feeding profile is applied to the process, i.e. mechanistic model based simulation.

Fig. 10 shows the actual biomass profile and the corresponding predictions from the aggregated ELM model. The poor performance obtained with the unreliable control policy (continuous blue line) can be seen from this figure, where the final biomass concentration value of $51.583[\mathrm{~g} / \mathrm{L}]$ is quite far away from the prediction by the aggregated ELM model $(\times)$ of 75.788 [g/L]. Conversely, with the feeding profile obtained when $\lambda=12$ (red dashed line), although the final value $71.236[\mathrm{~g} / \mathrm{L}]$ is not exactly the same as that predicted by the aggregated ELM model 75.409[g/L], the control policy is more reliable since it is shown to have good performance on the actual process. The small box at the top left of the graph is a zooming out window that shows closely the 
final value of biomass with the enhanced profile, and shows that the target without considering the confidence bounds $(X)$ was slightly higher than the target given by the reliable profile (ם).

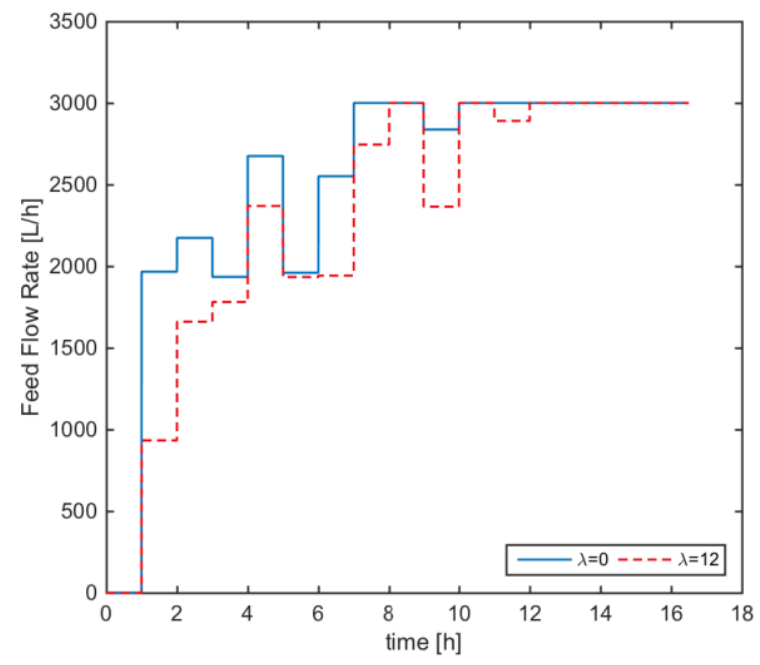

Fig. 9. Optimization results: control policy [18]

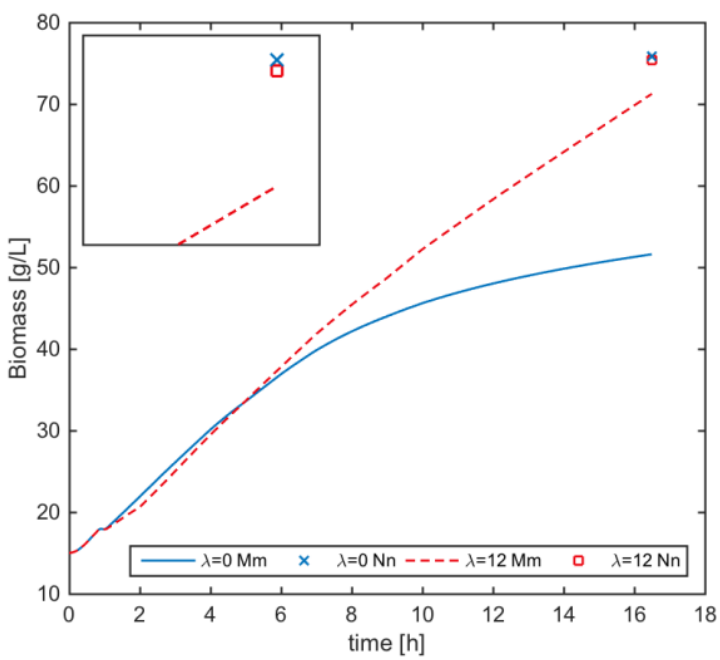

Fig. 10. Optimization results: biomass [18]

Fig. 11 shows the concentrations of glucose, oxygen, ethanol and the reaction volume during the batch. Particularly, it is interesting to analyze the ethanol formation, 
since is considerably high under the control policy with $\lambda=0$, with a final concentration around $30[\mathrm{~g} / \mathrm{L}]$, which is perhaps what is causing the drop of the final biomass concentration. It must be realized that Ethanol formation is undesirable, since it is a byproduct which can deteriorate the amount and quality of the product. On the other hand, the reliable control profile obtained with $\lambda=12$, gives better performance because the ethanol formation is successfully reduced to around $10[\mathrm{~g} / \mathrm{L}]$. Although this is not directly included in the objective function, it is implicitly related with the confidence bounds. In other words, when increasing the penalization of wide prediction confidence bounds, the optimization algorithm tries to find an optimal profile closer to the knowledge of the network, which was trained with data with reduced ethanol concentration. Therefore, the optimal control policy is more reliable also in the sense that it tries to generate a control policy that is well known by all the individual networks.
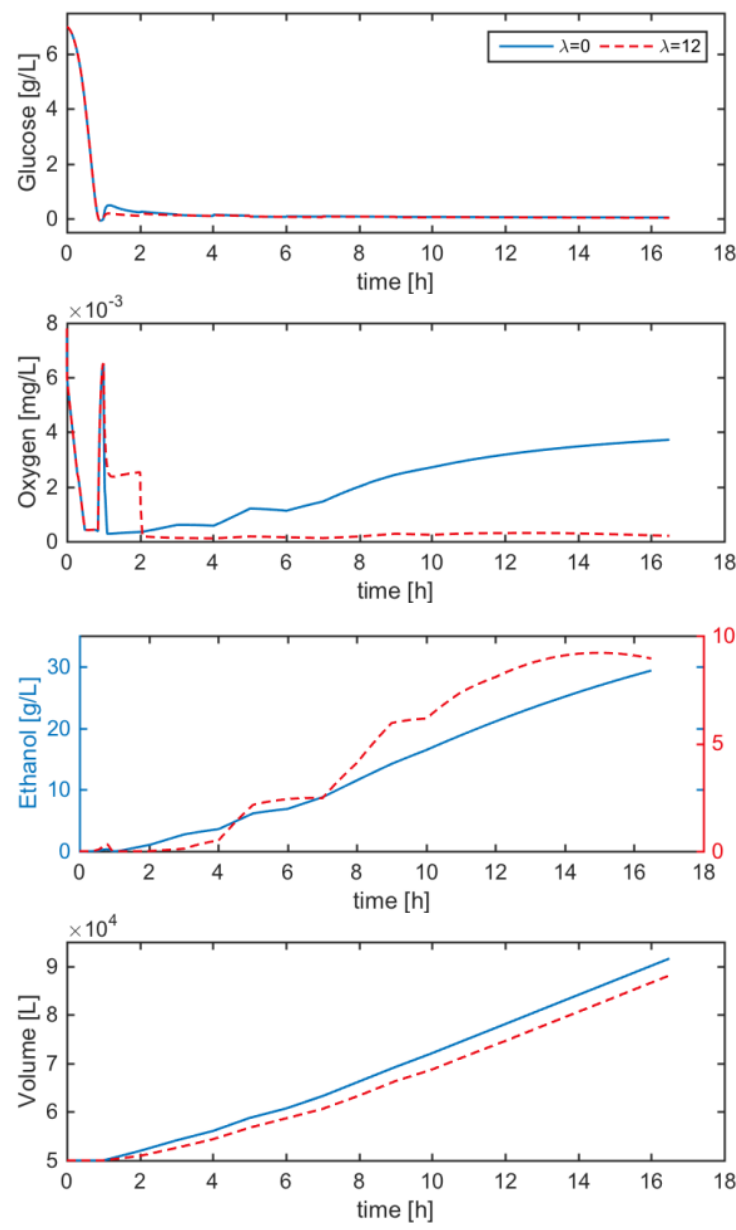

Fig. 11. Optimization results: glucose, oxy gen, ethanol, and volume [18] 


\subsection{On-line Re-optimization}

In addition to plant model mismatch, unknown disturbances can also lead to poor performance of the process. To cope with this situation, an on-line re-optimization strategy [12] is implemented by means of taking on-line measurements of the process every 4 hours and re-calculating the optimal control profile for the remaining batch period.

Initially, the optimal profile is calculated off-line for the entire batch time, $F_{0}=$ $\left[f_{1}, f_{2}, \ldots, f_{17}\right]$, with $\lambda=12$. The process is operated with the first two values $f_{1}$ and $f_{2}$ of $F_{0}$ being applied to the process (mechanistic model based simulation). Then, when two hours have elapsed, a measurement of the process is taken and, a new optimal profile is re-calculated for the remaining stages in the batch, which now starts from the third interval, and the result is given as $F_{1}=\left[f_{3}, f_{4}, \ldots, f_{17}\right]$. Note that the values in $F_{1}$ will generally be different from those in $F_{0}$. Then, the process is fed with the new $f_{3}, f_{4}, f_{5}$, and $f_{6}$, and 4 hours later the process is measured again. Similarly, a new optimal profile is estimated but now starting from the seventh interval $F_{2}=$ $\left[f_{7}, f_{8}, \ldots, f_{17}\right]$; just $f_{7}, f_{8}, f_{9}$, and $f_{10}$ are actually used because at the end of tenth interval the process is measured once again, and another re-optimized profile is found $F_{3}=$ $\left[f_{11}, f_{12}, \ldots, f_{17}\right]$; 4 hour later the last re-optimization is executed and the batch is finished with this profile $F_{4}=\left[f_{15}, f_{16}, f_{17}\right]$.

In order to perform the on-line re-optimization, it is necessary to develop four additional BA-ELM models that include as inputs the current process measurements and the feeding profile for the remaining batch period. All these models are aimed to predict the final biomass concentration at the end of the batch. Therefore, the new BA-ELM models can be written as follows:

- $\hat{y}=f_{N N 1}\left(C_{x}(2), F_{1}\right)$, where $C_{x}(2)$ is the biomass concentration measurement at $t=2 h$ and $F_{1}=\left[f_{3}, f_{4}, \ldots, f_{17}\right]$ are the feed flow rate intervals in $[L / h]$.

- $\hat{y}=f_{N N 2}\left(C_{x}(6), F_{2}\right)$, where $C_{x}(6)$ is the biomass concentration measurement at $t=6 h$ and $F_{2}=\left[f_{7}, f_{8}, \ldots, f_{17}\right]$ are the feed flow rate intervals in $[L / h]$.

- $\hat{y}=f_{N N 3}\left(C_{x}(10), F_{3}\right)$, where $C_{x}(10)$ is the biomass concentration measurement at $t=10 h$ and $F_{3}=\left[f_{11}, f_{12}, \ldots, f_{17}\right]$ are the feed flow rate intervals in $[L / h]$.

- $\hat{y}=f_{N N 4}\left(C_{x}(14), F_{4}\right)$, where $C_{x}(14)$ is the biomass concentration measurement at $t=14 h$ and $F_{4}=\left[f_{15}, f_{16}, f_{17}\right]$ are the feed flow rate intervals in $[L / h]$

The four BA-ELM models have a similar structure comprising 44 ELMs and the number of hidden nodes in each ELM is selected individually through cross validation. Fig. 12 shows the model performance of the four models for on-line re-optimization. It can be seen from Fig. 12 that all the four models give good prediction performance, especially $\mathrm{NN} 3$ and NN4 which also give very tight model prediction confidence bounds. This is expected as NN3 and NN4 use on-line measured process information closer to the end of batch.

Once the four BA-ELM models were developed, the process was simulated but a disturbance was introduced by modifying one of the mechanistic model parameters. The initial substrate concentration $S_{o}$ was change from its nominal value of $325 \mathrm{~g} \mathrm{~L}^{-1}$ 
to $305 \mathrm{~g} \mathrm{~L}^{-1}$, to pretend an unknown behavior of the process and validate the on-line optimization strategy.
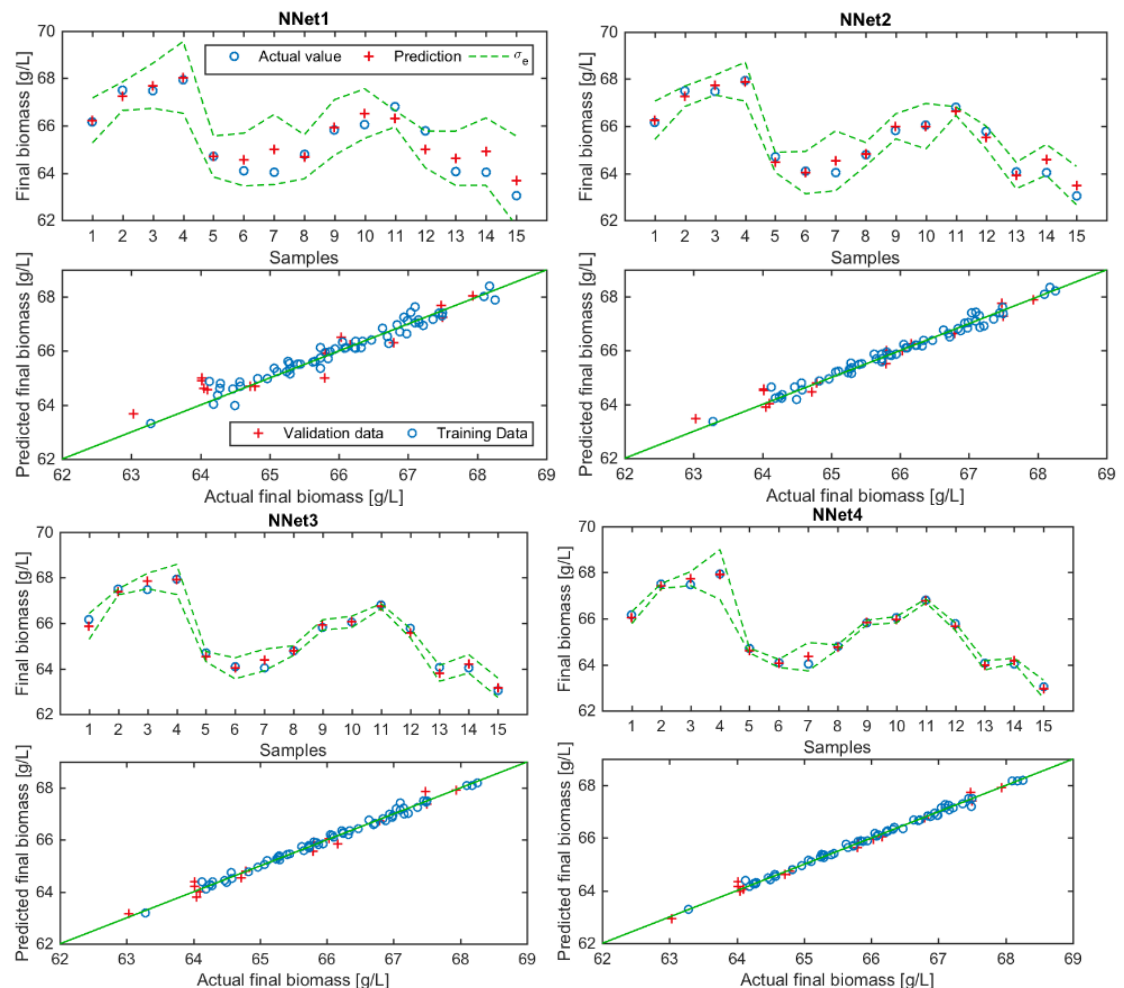

Fig. 12. On-line optimization results: control policy

Table 5. Final biomass concentration [18].

\begin{tabular}{lll}
\hline & Off-line & On-line \\
\hline Mechanistic model & 71.236 & - \\
Model + disturbance & 67.4971 & 71.1244 \\
Neural Network & 75.409 & 73.7741 \\
\hline
\end{tabular}

It can be seen from Table 5 that, by means of on-line updating the control policy, it is possible to compensate the initial deviation of the process due to the unknown disturbance, to achieve the same final biomass concentration as was obtained with the reliable off-line profile. The BA-ELM model prediction for the on-line case in Table 5 is given by the fourth neural network. Moreover, it is natural that, although the fourth neural network is the most accurate of all, an error between the actual process and the network prediction occurs, since the process is under the influence of the unknown disturbance and the BA-ELM model was not trained to learn any observation with that kind of mismatch. However, what is important rather than the error in the prediction is 
that the target final biomass was modified and reached a closer value to the desired target.

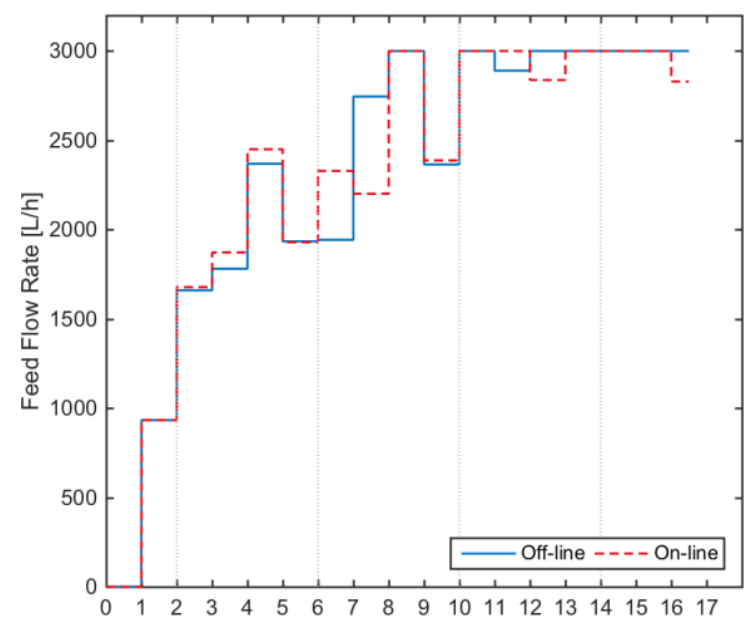

Fig. 13. On-line optimization results: control policy [18]

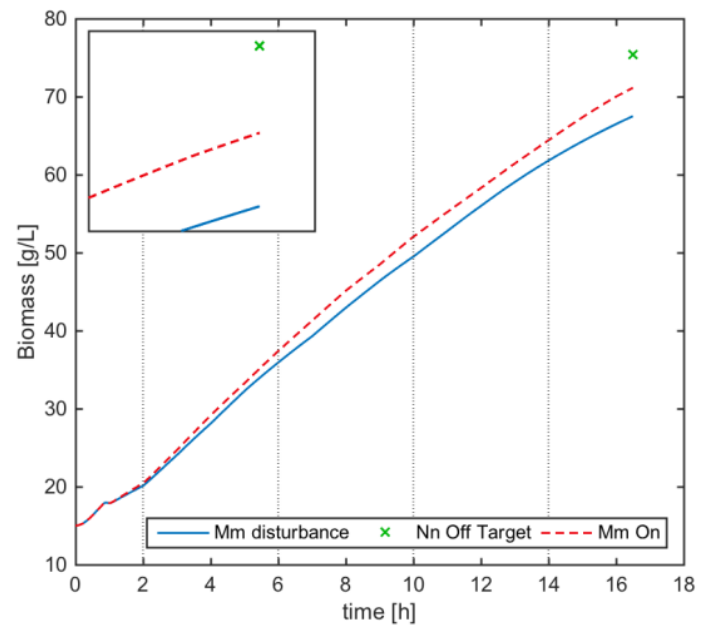

Fig. 14. On-line optimization results: biomass [18]

Fig. 13 shows the initial control policy calculated off-line (continuous blue line) and the re-optimized control profile (dashed red line) that was updated every four hours starting in the second hour, according to the division lines in the graph. Fig. 14 shows the biomas s concentration profile and it can be seen that during the first two hours both profiles are equal as no re-calculation has been performed. After the second hour, the feed flow rate is successfully modified through on-line re-optimization to drive the biomass concentration towards the desired optimal value. The small box at the top left 
corner is a zooming out window illustrating closely the difference between the off-line control policy applied on the process under disturbance (continuous blue line) and the on-line re-optimized profile (dashed red line).

Fig. 15 shows the profiles of the concentrations of glucose, oxygen, ethanol and volume profiles under on-line re-optimization. With respect to ethanol concentration, there is an extra amount of ethanolproduction, when the on-line re-optimization is performed, which perhaps is due to the efforts to achieve the biomass production target, since the substrate feed rate remains in the upper bound most of the time after the $10^{\text {th }}$ interval.
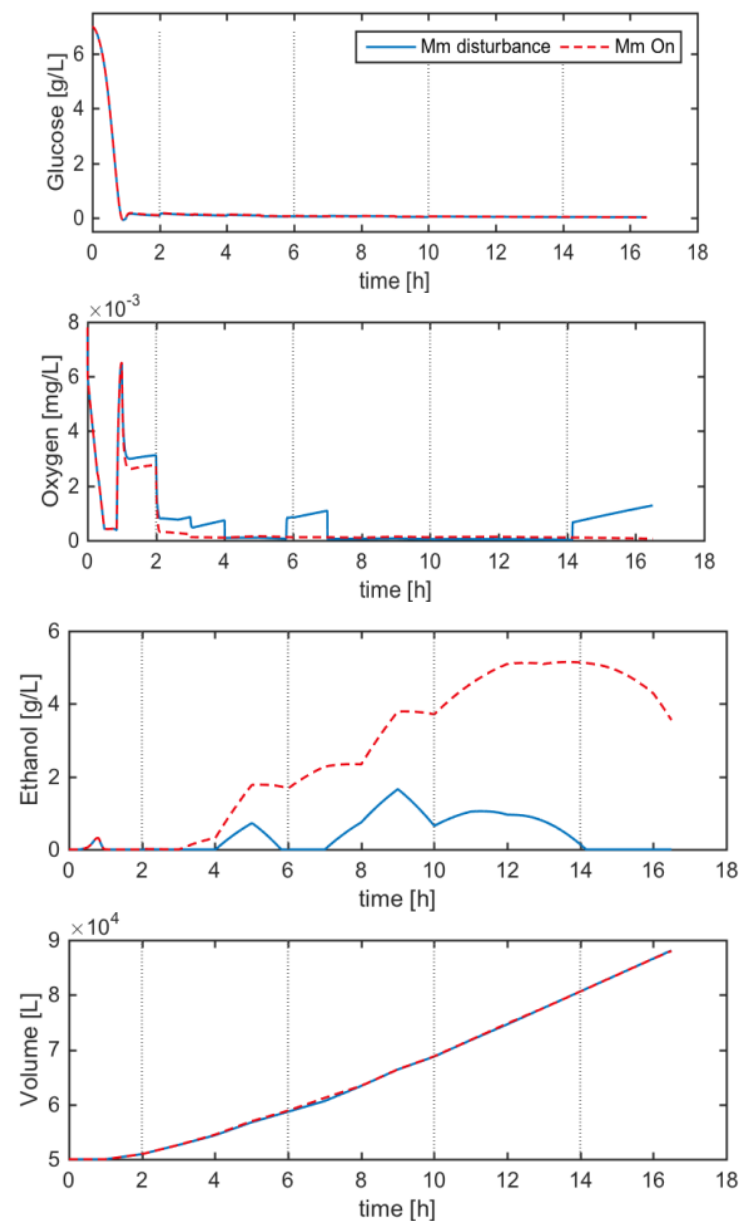

Fig. 15. On-line optimization results: glucose, oxy gen, ethanol, volume [18]

Finally, Fig. 16 shows all the re-optimized control profiles and highlights the time interval that is actually applied to the process with a thick line. For example, the offline control policy denoted as $F_{0}\left({ }^{-\cdot \cdot \cdot-}\right)$ is just applied for the first two hours, which 
are represented with a thick line. Then, after the second hour, the new optimal profile $F_{1}$ (一*-) is applied for the next four hours. Then, again a new re-calculation is made, and the profile $F_{2}(-\odot)$ is applied for four hours, when is replaced by $F_{3}(\longrightarrow \varkappa)$. The last re-optimization corresponds to $F_{4}(-\backsim)$, which is entirely applied, since it is the ending period of the batch. Consequently, although all the feeding profiles are calculated for the entire batch time, the resulting optimal profile, which is denoted as $F_{\text {final }}(----)$ is built just with the first four intervals of each profile.

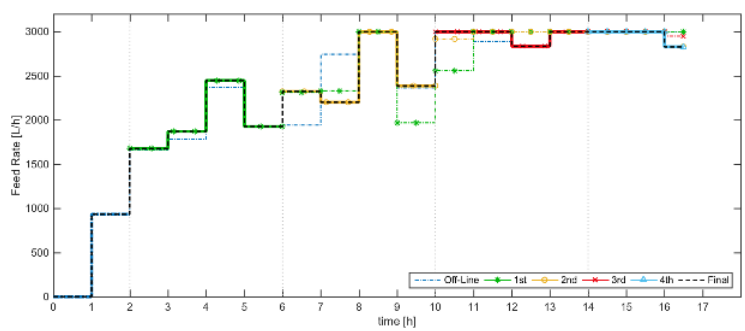

Fig. 16. On-line optimization results: detailed control policy [18]

\section{Conclusions}

Reliable modelling and on-line re-optimization control of a fed-batch fermentation process using bootstrap aggregated extreme learning machine (BA-ELM) is presented in this paper. It is shown that aggregating multiple ELM models can enhance model prediction performance. As the training of each ELM is very quick, building BA-ELM models does not introduce significant computation burden. Model prediction confidence bounds, a measure of model prediction reliability, can also be obtained for BA ELM. Minimizing the width of model prediction confidence bound is incorporated in the optimization objective so that the reliability of the calculated optimal control policy can be enhanced. In order to overcome the detrimental effect of unknown disturbances, on-line re-optimization is carried out to update the off-line calculated optimal control policy when the batch process is in progress. Applications to a simulated fed-batch fermentation process demonstrate the effectiveness of the proposed modelling and reliable optimization control technique.

\section{Acknowledgement}

The work was supported by the EU (Project No. PIRSES-GA-2013-612230) and National Natural Science Foundation of China (61673236). 


\section{References}

1. Benz, G.T.: Bioreactor Design for Chemical Engineers. CEP Magazine, August 2011, 21$26(2011)$

2. Chen, L., Zhang, J., Morris, A. J., Montague, G. A., Kent, C. A., Norton, J. P.: Combining neural networks with physical knowledge in modelling and state estimation of bioprocesses. In Proceedings of the 3rd European Control Conference, pp. 2426-2431, Rome, Italy, 5-8 September, (1995)

3. Tian, Y., Zhang, J., Morris J.: Optimal control of a fed-batch bioreactor based upon an augmented recurrent neural network model. Neurocomputing, 48(1-4), 919-936 (2002).

4. Tian, Y., Zhang, J., Morris, A. J.: Modelling and optimal control of a batch polymerisation reactor using a hybrid stacked recurrent neural network model. Industrial and Engineering Chemistry Research 40(21), 4525-4535 (2001).

5. Zhang, J., Morris, A. J., Martin, E. B., Kiparissides, C.: Inferential estimation of polymer quality using stacked neural networks. Computers \& Chemical Engineering, 21, s1025s1030 (1997).

6. Zhang, J., Morris, A. J.: Recurrent neuro-fuzzy networks for nonlinear process modelling. IEEE Transactions on Neural Networks, 10(2), 313-326 (1999).

7. Zhang, J.: Modelling and optimal control of batch processes using recurrent neuro-fuzzy networks. IEEE Transactions on Fuzzy Systems, 13(4), 417-427 (2005).

8. Noor, R. A. M., Ahmad, Z., Don, M. M., Uzir, M. H.: Modelling and control of different types of polymerization processes using neural networks technique: A review. The Canadian Journal of Chemical Engineering, 88, 1065-1084 (2010).

9. Huang, G.-B., Zhu, Q.-Y., Siew, C.-K.: Extreme learning machine: theory and applications. Neurocomputing, 70, 489-501 (2006).

10. Zhang, J.: Developing robust non-linear models through bootstrap aggregated neural networks. Neurocomputing, 25, 93-113 (1999).

11. Ahmad, Z., Zhang, J.: Combination of multiple neural networks using data fusion techniques for enhanced nonlinear process modelling. Computers \& Chemical Engineering, 30(2), 295308 (2006).

12. Xiong, Z., Zhang, J.: Neural network model-based on-line re-optimisation control of fedbatch processes using a modified iterative dynamic programming algorithm. Chemical Engineering and Processing: Process Intensification, 44, 477-484 (2005).

13. Xiong, Z., Zhang, J.: Optimal control of fed-batch processes based on multiple neural networks. Applied Intelligence, 22(2), 149-161 (2005).

14. Zhang, J., Jin, Q., Xu, Y. M.: Inferential estimation of poly mer melt index using sequentially trained bootstrap aggregated neural networks. Chemical Engineering and Technology, 29(4), 442-448 (2006)

15. Osuolale, F., Zhang, J.: Thermody namic optimization of atmospheric distillation unit. Computers \& Chemical Engineering, 103, 201-209 (2017).

16. Zhang, J.: A reliable neural network model based optimal control strategy for a batch polymerization reactor. Industrial and Engineering Chemistry Research, 43, 1030-038 (2004).

17. Yuzgec, U., Turker, M., Hocalar, A.: On-line evolutionary optimization of an industrial fedbatch yeast fermentation process. ISA Transactions, 48, 79-92 (2009).

18. Baron, C. M.C., Zhang, J.: Re-optimisation control of a fed-batch fermentation process using bootstrap aggregated extreme learning machine. In Proceedings of the 14th International Conference on Informatics in Control, Automation and Robotics (ICINCO2017), Madrid, Spain, 26-28 July (2017). 
19. Efron, B.: The Jacknife, the Bootstrap and Other Resampling Plans. Philadelphia: Society for Industrial and Applied Mathematics (1982).

20. Mukherjee, A., Zhang, J.: A reliable multi-objective control strategy for batch processes based on bootstrap aggregated neural network models. Journal of Process Control, 18(7-8), 720-734 (2008).

21. Herrera, F., Zhang, J.: Optimal control of fed-batch processes using particle swarm optimisation with stacked neural network models. Computers \& Chemical Engineering, 33(10), 1593-1601 (2009). 